\title{
Erratum to: Effects of Exposure to Domestic Physical Violence on Children's Behavior: a Chinese Community-based Sample
}

\author{
Yuping $\mathrm{Cao}^{1}$ • Longfei $\mathrm{Li}^{1,2}$ - Xingfu Zhao ${ }^{1,3} \cdot$ Yu Zhang $^{1}$ - Xiaoyun Guo ${ }^{4}$. \\ Yalin Zhang ${ }^{1}$. Xingguang Luo ${ }^{5}$
}

Published online: 5 May 2016

(C) Springer International Publishing 2016

\section{Erratum to: Journ Child Adol Trauma}

\section{DOI 10.1007/s40653-016-0092-1}

The acknowledgments that appear below are missing from the published article.

Acknowledgments This research was funded by the National Natural Science Foundation in China (Grant \#30670753), and American China Medical Board in New York, USA (Grant \#01749).

The online version of the original article can be found at http://dx.doi.org/ 10.1007/s40653-016-0092-1.

Yalin Zhang
zhangy169@vip.sina.com

1 Mental Health Institute, Second Xiangya Hospital, Central South University, Changsha, Hunan 410011, China

2 Jining Mental Hospital of Shandong Province, Jining, China

3 Mental Health Center, Wuxi, China

4 Shanghai Mental Health Center, Shanghai, China

5 Beijing Huilongguan Hospital, Beijing, China 